\title{
Cognitive training interventions for dementia and mild cognitive impairment in Parkinson's disease - A cochrane review summary with commentary
}

\author{
Tobias Loetscher
}

Cognitive Ageing and Impairment Neurosciences Laboratory, School of Psychology, Social Work and Social Policy, University of South Australia, Adelaide, Australia

E-mail:tobias.loetscher@unisa.edu.au.

\begin{abstract}
.
BACKGROUND: The majority of people living with Parkinson's disease will develop impairments in cognition. These impairments are associated with a reduced quality of life.

OBJECTIVE: The Cochrane Review aimed to investigate whether cognitive training improves cognition in people with Parkinson's disease and mild cognitive impairments or dementia.

METHODS: A Cochrane Review by Orgeta et al. was summarized with comments.

RESULTS: The review included seven studies with a total of 225 participants. There was no evidence for improvements in global cognition when cognitive training was compared to control conditions. Observed improvements in attention and verbal memory measures after cognitive training could not be confirmed in a subsequent sensitivity analysis. There was no evidence for benefits in other cognitive domains or quality of life measures. The certainty of the evidence was low for all comparisons.

CONCLUSIONS: The effectiveness of cognitive training for people with Parkinson's disease and cognitive impairments remains inconclusive. There is a pressing need for adequately powered trials with higher methodological quality.
\end{abstract}

Keywords: Parkinson's disease, cognitive training, training, dementia, mild cognitive impairment

The aim of this commentary is to discuss a rehabilitation perspective on the published Cochrane Review "Cognitive training interventions for dementia and mild cognitive impairment in Parkinson's disease" by Orgeta V, McDonald KR, Poliako E, Hindle JV, Clare L, Leroi I. ${ }^{1}$, under the direct supervision of Cochrane

\footnotetext{
${ }^{1}$ This summary is based on a Cochrane Review previously published in the Cochrane Database of Systematic Reviews 2020, Issue 2, Art. No.: CD011961, DOI: 10.1002/14651858.CD011961.pub2 (see www.cochranelibrary.com for information). Cochrane Reviews are regularly updated as new evidence emerges and in
}

Dementia and Cognitive Improvement Group. This Cochrane Corner is produced in agreement with NeuroRehabilitation by Cochrane Rehabilitation.

response to feedback, and Cochrane Database of Systematic Reviews should be consulted for the most recent version of the review.

The views expressed in the summary with commentary are those of the Cochrane Corner author and do not represent the Cochrane Library or Wiley. 


\section{Background}

Parkinson's disease (PD) is a progressive neurodegenerative disorder clinically defined by motor symptoms. The majority of people with Parkinson's disease will also develop impairments in cognition during the course of the disorder. At the time of PD diagnosis, approximately one out of four patients will meet the criteria of mild cognitive impairment (MCI). The prevalence of cognitive impairments does increase with age and time since diagnosis with the development of dementia being a likely consequence of PD.

Cognitive impairments are associated with a reduced quality of life, increased mortality and higher health-related costs. Treatment options for the cognitive impairments are needed to help PD patients maintain daily functioning and their quality of life. The benefits of pharmacological treatments for addressing cognitive impairments are limited. Cognitive training is a promising non-pharmacological intervention for cognitive impairments in a range of populations (Gavelin et al., 2020) and the goal of this Cochrane Review was to assess the efficacy of cognitive training in PD.

\section{Objective}

This Cochrane Review aimed to investigate the evidence for cognitive training interventions for dementia and mild cognitive impairment in Parkinson's disease (PD).

\subsection{What was studied and methods}

The population addressed in this review was people of all ages and settings with a diagnosis of PD and living with dementia or cognitive impairments. Of note, studies were also included if only a small proportion of study participants had cognitive impairments (e.g. in one study, only $23 \%$ of participants had mild cognitive impairment). The intervention studied was cognitive training - defined as any intervention involving the repeated practice of structured tasks that were developed to target domains of cognition. There were no restrictions regarding frequency, intensity and duration of training. Interventions could be conducted in individual or group settings. Multicomponent interventions were considered eligible if cognitive training was at least one of the components. Cognitive training was compared with an active control intervention (e.g., music, arts, motor rehabilitation) or no training (waiting list, usual care).

The primary outcomes studied were measures of global cognition and domains of cognition (executive function, attention, memory and visual processing). Secondary outcomes included measures of quality of life, activities of daily living and neuropsychiatric symptoms, carer burden, and adverse effects.

A systematic search of randomized controlled trials (RCTs) was conducted up to August 8, 2019. The search involved a specialized Cochrane register (ALOIS) maintained by Cochrane's Dementia and Cognitive Improvement Group, electronic databases and trial registers.

\section{Results}

The review included seven studies with a total of 225 participants. All interventions were delivered by computer for four to eight weeks in duration. There was a high level of adherence in the five studies that reported information about adherence to the intervention. All studies assessed the outcomes at the end of the intervention. The review shows that when compared with a control group, there is:

- no evidence for a cognitive training effect on global cognition (6 studies, 178 participants)

- slightly better performance in attention and verbal memory measures after cognitive training (5 studies, 160 participants). However, evidence for such improvements was not found in a subsequent sensitivity analysis in which a study comprising mostly people without cognitive impairment was excluded.

- no evidence for cognitive training effects on other cognitive domains (executive functioning, visual processing), activities of daily living and quality of life. One study reported no adverse effects, and all other studies did not report any information about adverse effects.

Two studies with a total of 41 participants did also conduct follow-up assessments at 12 and 24 weeks, respectively. There was no evidence for any benefits of cognitive training at the follow-up assessments.

The certainty of the evidence (GRADE) was judged to be low for all comparisons - meaning that the reported effects may substantially change if additional studies become available. 


\section{Conclusions}

The authors concluded that there was no evidence for important cognitive improvements deriving from cognitive training. They cautioned that their conclusions were based on a small evidence base, studies with methodological limitations and low certainty of the overall evidence. They call for more robust studies assessing the efficacy of cognitive training in people with Parkinson's disease.

\subsection{Implications for practice in neurorehabilitation}

The efficacy of cognitive training for people with Parkinson's disease and living with cognitive impairment remains inconclusive. While there were some encouraging findings such as beneficial effects on attention and verbal memory, these benefits remain highly uncertain due to the small number of studies and their methodological quality.

Clinicians and researchers are currently unable to provide advice on the efficacy of cognitive training approaches for people with PD and cognitive impairment. Given some promising evidence for the efficacy of cognitive training in older people with cognitive impairment but no PD (Hill et al., 2017), in people with PD without dementia (Leung et al., 2015), and the lack of highly effective pharmacological treatment for cognitive impairment in PD, there is a critical need for robust and adequately powered randomized controlled trials to assess the efficacy of cognitive training in PD. For important methodological considerations when planning a robust cognitive training study in people with PD see Walton et al. (2017).

\section{Acknowledgments}

The author thanks Cochrane Rehabilitation and Cochrane Dementia and Cognitive Improvement Group for reviewing the contents of the Cochrane Corner. He was supported by a National Health and Medical Research Council (NHMRC) Dementia Research Leadership Fellowship (GNT1136269).

\section{Conflict of interest}

The author declares no conflicts of interest.

\section{References}

Gavelin, H. M., Lampit, A., Hallock, H., Sabatés, J., \& BaharFuchs, A. (2020). Cognition-oriented treatments for older adults: A systematic overview of systematic reviews. Neuropsychology Review, 30, 167-193.

Hill, N. T., Mowszowski, L., Naismith, S. L., Chadwick, V. L., Valenzuela, M., \& Lampit, A. (2017). Computerized cognitive training in older adults with mild cognitive impairment or dementia: a systematic review and meta-analysis. American Journal of Psychiatry, 174(4), 329-340.

Leung, I.H., Walton, C.C., Hallock, H., Lewis, S.J., Valenzuela, M., \& Lampit, A. (2015). Cognitive training in Parkinson disease: a systematic review and meta-analysis. Neurology, 85(21), 18431851.

Orgeta, V., McDonald, K. R., Poliakoff, E., Hindle, J. V., Clare, L., \& Leroi, I. (2020). Cognitive training interventions for dementia and mild cognitive impairment in Parkinson's disease. Cochrane Database of Systematic Reviews, Issue 2, Art. No.: CD011961

Walton, C. C., Naismith, S. L., Lampit, A., Mowszowski, L., \& Lewis, S. J. (2017). Cognitive training in Parkinson's disease: a theoretical perspective. Neurorehabilitation and Neural Repair, 31(3), 207-216. 